\title{
Knitting the Comparative Case Study (CCS) with Mixed Methods: An Attempt to Extend the Methodological Application of CCS
}

This paper offers a critical analysis of the Comparative Case Study (CCS). While CCS provides a holistic methodological framework for case-based research, it is not free from drawbacks. One criticism the approach may receive, ironically, is the lack of criticism it encounters. Another critique can include its naming, as the name 'comparative case study' may not sufficiently represent its vital feature of transversal, vertical and horizontal axes. Lastly, the paper examines the qualitative approach traditionally associated with CCS deriving from its roots in anthropological and ethnography-oriented concepts and methods. We argue that the use of mixed methods can strengthen the CCS approach. This article is intended to stretch out the applicability of CCS through an empirical example with mixed methods on learner-centred pedagogy in Tanzanian primary schools.

Keywords: comparative case study; vertical case study; mixed methods; learnercentred pedagogy; Tanzania

\section{Introduction}

Developed by Vavrus and Bartlett (2006, 2009; Bartlett and Vavrus 2014, 2017), the Comparative Case Study (CCS) approach provides a methodological framework to study how policies are formed and adapted across time in different settings and scales. It inquires into how history, sociocultural settings, and national and international political structures influence policy appropriation, using three methodological axes: the horizontal, the vertical and the transversal axes (Figure 1). The horizontal axis denotes multi-sited comparisons of policy implementation. It assesses how differently and similarly each case within distinctive 
localities contextualises and appropriates certain policies at a given point in time. Situating the horizontal cases within their cultural and social network, the vertical axis analyses how policy discourse within and between global and national institutions shape the social norms and practices of individual units of analysis, such as schools. The transversal axis contextualises the horizontal and vertical components in the past and present of broader social settings. It traces how the data from different physical places (horizontal axis) and different policy levels (vertical axis) intersect with, and are influenced by, historical contingencies. While the CCS provides a comprehensive methodological framework for case study research, it is not however free from drawbacks. By providing a critical examination of the CCS, this paper intends to further enhance the methodological rigour of this approach.

(Insert Figure 1.)

One criticism the CCS may receive, ironically, is the lack of criticism it encounters. Various researchers have adopted the CCS model for their multi-sited case studies (e.g. Bellino 2016; Gardinier 2015). However, they have seldom considered the limitations of the framework, merely adopting the transversal, vertical and horizontal axes as appropriate methodological tools. There is barely any research, to our knowledge, that has judiciously analysed, applied and refined the CCS approach. This in itself can be a weakness of this methodology, given the lack of academic scrutiny. Analytical engagement with the CCS framework would strengthen this methodological tool.

Another critique concerns its naming. Vavrus and Bartlett have revised the naming of the framework as they modify and enhance the concepts underpinning the CCS. The framework was born with vertical and horizontal axes as primary interests, labelled the ‘vertical case study' (Vavrus and Bartlett 2006, 2009). Although the authors insinuated 
inclusion of transversal tracing (Vavrus and Bartlett 2009, 15), the historical dimension had not gained significant attention until a later conceptualisation. Bartlett and Vavrus (2014) introduced the transversal comparison as the third axis that locates policy appropriation within a historical milieu. The modification of the framework nevertheless did not lead to altering its name. Despite the equivalent importance placed on the three axes - the transversal, vertical and horizontal - the term 'vertical case study' remained as if it prioritised the vertical axis.

Upon reflection, Bartlett and Vavrus (2017) consolidated a decade of their work and renamed the framework as a 'comparative case study'. Even though this term may denote equal value placed upon the three axes, it suggests comparison between rather than the intersection of the three axes, and is also too generalised and might miss the significance of the axes. Any case study - whether it applies the three axes or not - can be comparative. Yin (2014) distinguishes two approaches used in a case study: single- and multiple-case studies. The latter is commonly called ‘comparative case study' (e.g. Sheridan et al. 2014; MonteSano 2008). These studies often present comparisons between cases, albeit comparing only horizontal cases in Bartlett and Vavrus's model. A more nuanced naming of the CCS could have shed more light on the unique features of the transversal, vertical and horizontal axes to differentiate their comparative case study from others.

Further limitation of the CCS pertains to its methodological application. Derived from the anthropological tradition that Bartlett and Vavrus come from, the CCS endorses ethnography-oriented research. It is grounded in interpretivist, constructivist epistemology which employs qualitative methods (Bartlett and Vavrus 2017). The researchers unequivocally criticise variable-oriented, quantitative research for its ignorance of context, unpredictability and the co-constructedness of human behaviour. At the same time, Bartlett and Vavrus (2017) appear to appreciate the usefulness of mixed methods: 
[W]e assume that most readers intend to use primarily qualitative research methods, such as observation, interviewing, and discourse analysis, but we also emphasize that questionnaires or surveys can help to gain a comparative perspective. (7)

The authors introduce in detail the survey method for collecting quantitative and qualitative data to help investigate what is represented by the three axes (Bartlett and Vavrus 2017). Nonetheless, there is little clarification as to how a researcher can utilise and incorporate a quantitative strand with positivist epistemology into the CCS approach; this is reflected in the much smaller number of mixed methods approaches in existing CCS research compared to qualitative studies. Even when some researchers used quantitative and qualitative methods, their research could be described as qualitative studies with an addition of quantitative data rather than mixed methods research with 'a specific research design that includes rigorous, systematic and the planned use of different quantitative and qualitative methods for collecting and/or analysing data in the same study' (Cara 2017, 195).

For example, Shriberg (2008) describes her vertical case study in Liberia as mixed methods research, but with little attention to the above criteria. The study examined how the policy intentions of international and national government affected teachers' well-being. Along the horizontal axis, the researcher conducted interviews with teachers and policymakers and participant observations in primary, secondary and university classes. Shriberg also distributed a survey to teachers as a quantitative data technique, but the research was designed not as 'mixed methods research' but more as a qualitative study aided by the survey. The author seldom discusses the issues crucial to mixed methods research, such as: mixing of ontological and epistemological stances; the relative priority given to the 
two data types; the level and timing of mixing quantitative and qualitative datasets; and integrating quantitative and qualitative findings (Creswell and Plano Clark 2011). As a result, quantitative analysis only presented descriptive statistics and frequencies of certain responses. More rigorous data analysis - such as data transformation, correlation, comparison and/or integration (Onwuegbuzie, Johnson, and Collins 2009) - would have corroborated her findings.

Given the lack of explanation as to the relevance or irrelevance of mixed methods within the CCS approach, the paper now turns to an empirical example. Integrating quantitative components with a qualitative case study would not harm but add richness to the data. Following a brief introduction of the research on the implementation of learner-centred pedagogy (LCP) in Tanzanian primary schools, we elucidate the strengths of mixed methods and the possibility of marrying it with the CCS approach. We then present results from quantitative and qualitative datasets while highlighting advantages of mixed methods.

\section{The research}

The research aimed to investigate how primary schools in Tanzania have conceptualised and implemented LCP and whether and how LCP may contribute to pupil learning. Although the literature consistently suggests the incompatibility and challenges which plague LCP implementation in low-income nations (e.g. Schweisfurth 2011; Vavrus 2009), understudied areas still remain. In spite of the similarities between learner-centred ideas and educational philosophy and policies historically developed in Tanzania (Nyerere 1967, 2004; Author 2), little research conducted in the country has thoroughly conceptualised findings in terms of its historical/ideological settings, although some researchers embed their empirical results within Tanzania's socio-cultural settings (e.g. Barrett, 2007; Vavrus, 2009). Additionally, not only in Tanzania but also in other low-income countries, studies on LCP implementation have 
prevalently centred on teachers, exploring their beliefs about teaching and learning and their act of teaching; student perspectives on LCP implementation have attracted much less scholarly attention (Tabulawa 2003, 2013; Schweisfurth 2011). Furthermore, hardly any research has considered the processes and likely improvements that can emerge from LCP implementation, despite the belief that LCP can lead to better learner outcomes (McCombs and Miller 2007; Cornelius-White and Harbaugh 2010). Another aspect indicating the knowledge gap with respect to LCP in existing literature concerns methodology. Among the studies reviewed during this research, the majority (40 out of 65) used mainly qualitative methods, compared to 17 and 8 respectively applying quantitative (e.g. Author 2; Hardman, Abd-Kadir, and Tibuhinda 2012) and mixed methods (e.g. Sifuna and Kaime 2007). Even though the review was not a systematic review but was conducted narratively, our claim about the paucity of certain methodology mirrors the views of Schweisfurth (2011) and Frost and Little (2014) that large-scale, quantitative studies on pedagogy are lacking in low-income countries. Given these gaps in knowledge, the research questions asked: 1) How is Tanzania implementing LCP in primary schools given the historical/ideological context of the country's education system and provision?; and 2) Is observed LCP implementation associated with pupils' perceptions of classroom experiences and/or their learning outcomes? If so, which specific pupil perspectives relate to LCP implementation?

\section{Research design: Transversal and vertical investigations}

The transversal and vertical examinations in this research were conducted through a literature review and document analysis, and then through a comparison of these with interview accounts from 17 present teachers and a son of the first president Nyerere, Mr Madaraka Nyerere recognising his expertise in President Nyerere's policies. The research first charted education from the indigenous period to the postcolonial era, with a greater focus on 
Nyerere's seminal policy, Education for Self-Reliance (ESR). ESR promoted similar concepts of LCP, such as hands-on learning and democratic teacher-student relationships (Nyerere 1967). In her interview with Nyerere's son, author 1 asked about what Nyerere thought of these dimensions of ESR. The historical review of the literature and the narratives of Mr Madaraka Nyerere were compared with the present teachers' accounts gained from semi-structured interviews, exploring whether and how Nyerere's philosophy influenced the pedagogical approach of the present-day teachers.

As the transversal review moves from indigenous period to more recent times, the transversal axis eventually meets the vertical axis. By exploring the history of international policy development, the research considered how the Western-born LCP has been spreading to non-Western countries over past decades (Tabulawa 2003; Mundy et al. 2016). It inquired into how the development agenda and donor pressure affects the formation of Tanzanian national education policies. This aimed to deconstruct the hegemonic influences of global education forces in the national policy formation processes.

\section{Horizontal comparison with mixed methods}

Along the horizontal axis of the CCS, this research utilised a total of six methods of qualitative and quantitative nature within a qualitative-led, embedded mixed methods research design. The reasons for using mixed methods were threefold: the value of two contrasting paradigms; several strengths of mixed methods; and an attempt to make a methodological contribution.

Pertaining to the first point, the research appreciated the value of different paradigms by adopting a dialectical perspective. The dialectical paradigmatic view argues that an effort to overcome a conflict or contradiction by means of a dialogue between contrasting paradigms can generate a better understanding of data (Greene, Benjamin, and Goodyear 
2001). To justify the use of constructivism in this research, this view proposes that reality is formed culturally and socially and that no singular reality exists (Patton 2015). Classroom reality is constructed and negotiated between teachers and students through interaction in a particular social context at a particular time. Acknowledging this understanding of pedagogy requires an epistemology that recognises the roles played by culture, social structure and human agency in constructing students' knowledge. Furthermore, the ontological relativism featured in constructivism values the multiple realities experienced by different groups (Patton 2015). Teachers and students participate in the co-construction of knowledge, reality, meanings and relationships; but each party may perceive and understand the co-constructed truth differently. This is of particular interest in this study to address the lack of children's voices in the previous literature on LCP implementation in low- and middle-income countries (Tabulawa 2013; Schweisfurth 2011). Although constructivism acts as the primary paradigm, the research also integrated the quantitative approach associated with positivism. The statistical analyses tested the relationships between LCP and pupil learning to draw an inference as to whether LCP yields better learning outcomes. Following the dialectical manner of mixing paradigms, the research valued both ways of knowing, where the constructivist paradigm provided the epistemological and ontological lenses to explore how different groups of people understand and practise LCP whilst the positivist paradigm played a role in supporting the qualitative findings.

In addition, mixed methods were chosen in relation to their strengths. Firstly, mixed methods can answer research questions that may not be addressed by either quantitative or qualitative methods alone. The former method generally undertakes confirmatory research questions to test a theory, whereas the latter engages with exploratory research questions to generate a theory (Tashakkori and Teddlie 2003). What shall we do if we wish to simultaneously answer confirmatory and exploratory research questions, such as the case of 
this research? The question about how and why the ideological and historical background of Tanzania affects its appropriation of globalised LCP policies requires an in-depth description of cultural phenomena to be explored through qualitative approaches. The other research question inquired about the statistical relations between the observed level of LCP implementation, pupils' views of LCP, and their learning outcomes. Specifically, the research investigated whether pupils' academic performance and learning attitudes vary between schools, and if so, whether the variation is associated with the levels and consistency of LCP implementation in their classrooms. Mixed methods enabled the research to investigate both exploratory questions for theory generation and confirmatory questions for theory verification in a single study.

In addition, triangulating methodologies can corroborate research validity by offsetting the weaknesses of qualitative or quantitative methods when employed alone (Greene, Caracelli, and Graham 1989). Quantitative research is also criticised for its ignorance of contexts and tendency to assume the universal applicability of their findings or the tested theory (Creswell and Plano Clark 2011). Qualitative studies tackle these problems, but they sometimes face accusations relating to their subjective choices and interpretations. The small number of participants also makes it difficult to apply the results to other groups. This study aimed to minimise these challenges by bringing together multiple sources of data. Moreover, mixed methods allow researchers to elaborate and illustrate statistical results with narrative details (Greene, Caracelli, and Graham 1989). Structured lesson observations and questionnaires looked into variables influencing LCP implementation and pupil perceptions in a quantifiable way. Interviews and focus group discussions (FGDs) added narrative elements to the numerical evidence, elaborating how and why the quantitative variables of LCP implementation are observed in different schooling conditions. 
Lastly, the relative lack of mixed methods studies on LCP implementation motivates its use. Schweisfurth (2011) and Frost and Little (2014) cast doubt on potential methodological imbalance in the studies on LCP implementation in developing countries. As discussed above, the research also addresses the relative shortage of mixed methods studies using the CCS approach. Similar results arising from methodologically-contrasting studies can substantiate each other (Firestone 1987). An addition of mixed methods research may thus contribute to methodological triangulation for the literature.

The above accounts demonstrate the advantages of mixed methods, but mixed methods research also entails several challenges. One of the most notable questions arises from mixing epistemologies from the quantitative and qualitative approaches. This is exemplified by a heated 'paradigm war' since the late 1980s (Tashakkori and Teddlie 2003). Inconsistency between qualitative and quantitative datasets may also result in challenges in meeting the quality criteria. Another point to consider concerns feasibility and practicality. Creswell and Plano Clark (2011) warn that conducting rigorous mixed methods research requires extensive research skills, time and resources. It can also demand more human and material resources, posing the question of practicability. Although mixed methods research entails shortcomings in these respects, if carried out adequately, triangulation helps corroborate the findings of qualitative and quantitative methods.

With the mixed methods methodology applied within the horizontal axis of the CCS, the study employed a qualitative-led, embedded mixed-methods research design. The qualitative approach guides the overall research design, with a supportive role played by the quantitative approach (Creswell and Plano Clark 2011). The philosophical assumption in this design depends on the primary approach, or the qualitative strand within the constructivist paradigm in the case of this study. 
Within the overarching CCS approach with mixed methods, the embedded multiplecase design (Yin 2014) guided the case selection process. The selection criteria included location (urban or rural) and school types (public or private). The combination of these criteria produced four categories of urban public, urban private, rural public and rural private schools, although at the analysis phase private schools were combined into one category regardless of location because both urban private and rural private schools exhibited similar characteristics. Based on the national examination results, the research first selected two regions of Dar es Salaam and Kigoma, in which schools were purposefully selected based on the above selection criteria. A total of 13 primary schools - in which 1,024 pupils of Standard 6, 17 teachers and 13 head teachers were situated - participated in this study.

In English or maths classes at the 13 schools, author 1 engaged in participant observation in the 17 classes by taking memos of what she saw, thought and experienced with regards to LCP. The lessons were also video-recorded for systematic analysis with observation schedules. This structured classroom observation quantitatively measured the time spent on learner-centred, teacher-centred and off-task activities in one lesson. The activities were classified into these three categories based on studies previously conducted with similar research focus. LCP-related activities included: individualised activities (Frost and Little 2014); group work (Frost and Little 2014; Ackers and Hardman 2001); pupil demonstration (Ackers and Hardman 2001); and learner-initiated questions and answers (Q\&A) (Hardman, Abd-Kadir, and Smith 2008; Pontefract and Hardman 2005). Teachercentred tasks were: watching/listening (Frost and Little 2014); taking notes (Frost and Little 2014); reading aloud (Ackers and Hardman 2001); writing exercises (Ackers and Hardman 2001); and teacher-initiated Q\&A (Ackers and Hardman 2001; Hardman et al. 2008; Pontefract and Hardman 2005). Lastly, off-task activities involved: teacher management (Frost and Little 2014); transition (Author 2); and pupil uninvolved (Frost and Little 2014). It 
should be acknowledged that the observation schedule built on literature published in the Global North; the understandings of the categories might well be different from Tanzanian understandings of learner-centred, teacher-centred or off-task activities. Another concern of structured observation involves the observer effects. The mere presence of the researchers, especially when they are unfamiliar to the researched population, such as in the case of this study, is likely to affect and change participant behaviours (Robson 2002; Bryman 2016).

Additionally, author 1 invited the 17 teachers to semi-structured interviews. The interviews sought to substantiate the answers to how LCP is actualised in schools and which teacher characteristics may or may not relate to appropriation of the pedagogy. The interviews also investigated Tanzania's ideological compatibility with LCP by enquiring into present-day views of the historical figure of Julius Nyerere. With three girls and three boys in the 17 observed classes, author 1 conducted FGDs to examine their perceptions and experiences of schooling and classroom pedagogy. She asked about which classroom activities they preferred and which less so, what they would like to change in their classes and/or school, and how they interacted with their family members. These questions explored to what extent Tanzanian culture appreciates child-centredness beyond school settings, which may have implications for pupil-teacher relationships in school.

Moreover, the head teachers $(\mathrm{N}=13)$, teachers $(\mathrm{N}=17)$ and pupils $(\mathrm{N}=1,024)$ filled out researcher-administered questionnaires. The head teacher and teacher questionnaires sought their academic and professional backgrounds, resource availability, and their views toward school culture and pupils. In the pupil questionnaire, the respondents provided their socioeconomic and family backgrounds. It then explored the pupils' subjective perceptions of LCP implementation, which was denoted as 'perceived-LCP' as opposed to LCP-related activities observed through structured and unstructured observations. The pupils rated on a five-point Likert-scale how much they felt they were engaged in LCP-related classroom 
practices (Knight et al. 2014). The questionnaire for pupils also investigated their learning attitudes to measure one aspect of learning outcomes. Finally, to assess if LCP can improve pupil learning, the pupils were asked to complete an exam (English or mathematics). The exam was a modified version based on Tanzania's official exams, used in one of the local government areas visited in the research. The Table below summarises the achieved sample for each data generating method.

(Insert Table 1.)

Integrating the transversal, vertical and horizontal axes, Figure 1 visualises the overall CCS framework applied to this research.

(Insert Figure 2.)

At the analysis stage, the horizontal comparison followed the principle of the dialectical philosophical assumption to appreciate multiple views of knowledge. By moving back and forth between qualitative and quantitative datasets, data analysis engaged in what Greene, Benjamin and Goodyear (2001) call 'a “conversation” between different paradigms' (28). Whenever contradictive results derived from different philosophical stances were found, the analysis sought to provide possible explanations as to why this occurred. Sometimes they seemed to result from participants' social positions (i.e. pupils, teachers or researcher); at other times different methods might have produced contrasting results. The findings below aim to demonstrate that the integration of data representing different philosophical assumptions can contribute to more relevant and comprehensive inferences. 


\section{Mixed methods findings within the CCS framework}

The transversal, vertical and horizontal axes explored the traveling feature of LCP across time and space, with respect to various policy levels and localities. Arranged by the three axes of the CCS and different methods used, Table 2 presents which axis examined which pedagogical dimensions through which methods.

(Insert Table 2.)

\section{Transversal and vertical exploration}

Attending to the historical facets of pedagogy, the transversal axis explored the educational development of Tanzania, which appeared on the surface somewhat compatible with the LCP tenets. Based on the view that knowledge is a product of social construction, LCP is often characterised by: attention to individuals (Rousseau 2007); learner independence (Brandes and Ginnis 1986); democratic pupil-teacher relationships (Dewey 1916); active roles played by learners; and collaborations and interactions (Vygotsky 1986). Such principles of LCP arguably form a common ground with the educational agenda historically fostered by Julius Nyerere in Tanzania. The president endeavoured to break down boundaries between schools and communities and to make curricula relevant to everyday life (Nyerere 1967). Discussions and decision-making exercises to practice democracy at school also topped his educational agenda (Nyerere 2004):

[An] essential part of the success of our attempt to build a democratic society is the combination of free discussion followed by the full implementation of joint decisions; if the children get used to this at school they will at the same time be learning about the responsibilities of citizens in a free society. (93) 
How does this seemingly-consonant ideological base play out in the midst of international and national efforts to implement LCP today? The vertical investigation explored policy diffusion processes of LCP tenets throughout developing countries. Since the launch of Education for All in 1990, terms such as 'participatory methods' (UNESCO 2017, 18) and 'child-centred teaching and learning processes' (UNICEF EAPRO 2006, 18) have gained popularity in policy documents. This vertical analysis recognised a narrow conceptualisation of pedagogy in the international policy documents as relating it only to the observable act of teaching. They outline how teachers should act, or at best how they should be trained. For instance, UNESCO (2007) introduces the 'child-centred curricula' and discusses 'a move away from "chalk and talk" methods to more discovery-based learning' (29). It implicitly equates curricula with a teaching technique. UNICEF's child-friendly schools appear to adopt a holistic approach involving school design, community involvement and children's rights; but its pedagogical focus attends to teachers and teaching methods, mostly considering how better to train teachers to use a child-centred approach (UNICEF 2009). Few accounts are made of learning, learners, cultural values, pupil-teacher relationships, and so on. The Tanzanian government, as the recipient of these verticallytransmitted global policies, pursues the international recommendations in its national agenda, with a prioritised focus on observable LCP practices. This tendency of international and national policymaking to reduce pedagogy to a mere teaching method may reflect their implicit assumption that as long as LCP-related activities were observed in the classroom, LCP implementation would be successful.

The transversal and vertical axes have examined the historical, social and cultural milieu Tanzania has nurtured across time and space. Below the horizontal inquiries will 
investigate whether and how they comes out as a practice and how they related to pupils' schooling experiences and learning outcomes.

\section{Horizontal investigation (1): Classroom observation}

The mixed methods results along the horizontal axis revealed rather contradictory results with these policy expectations transversally and vertically diffused. A collective average of 17 lessons showed that teacher-centred activities dominated the lesson time (81\%) across the 13 participating schools, whereas the teachers spent $14 \%$ on learner-centred activities. Offtask activities took up 5\%. The pupils mostly watched and listened to the teachers and were independently engaged in writing exercises, and no activity adjusted to individual pupils took place. We acknowledge the limitation of such an approach to define and categorise the types of activities based on Northern criteria, which might differ from the local understandings of LCP.

However, a systematic counting of activities and interactions sometimes violate construct validity of the observation data, and a combination of methods can facilitate crossvalidation of the findings and conclusions. Known as the observer effect, the mere presence of the researchers may well stimulate and alter certain behaviours (Bryman 2016).

Complementing the validity of structured observation, unstructured observation allowed a transparent report of what was happening. For example, there were instances where teachers might have practised LCP-related activities to set up their classes to 'put on a show' for the sake of this research. At Amani School, Juma directed around 50 pupils to form small groups. By repeating 'Class, quiet, quiet', the teacher gave an instruction to silently read the sentences he drew on the blackboard. Few verbal discussions took place, and being in a group meant little for the pupils. In a mathematic lesson led by Chane, group presentation - another LCP-associated observable activity - took place. Using a wooden stick, one representative 
from selected five groups indicated points A to $\mathrm{G}$ on the coordinate geometry drawn on the front board:

Girl: $\quad$ A, we have got positive four.

Chane: Where do you get positive four? Where do you get positive four? At which axis?

Girl: $\quad$ X.

Chane: $\quad$ Okay, $\mathrm{X}$ axis. Is it right?

All pupils: She is right.

Girl: $\quad$ B... B, we get positive two at $\mathrm{X}$ axis, and we've got negative four at $\mathrm{Y}$.

Because all groups solved the same questions, all presenters repeated the same answers in a similar manner as above. Toward the end of the lesson, the non-presenting pupils seemed to be bored of the repetitive activity. Whilst we should be careful not to dichotomise classroom activities into learner-centred or teacher-centred pedagogies because in reality classroom practices involve a mixture of both techniques and ideas (Guthrie 2018; Schweisfurth 2013), the above portrayals - enabled by detailed note-taking during unstructured observations - exemplify that some teachers seemed to apply surface features of observable teaching techniques but without engaging with LCP principles. Mtika and Gates $(2010,400)$ also came across such an instance in Malawi. Their observation note states that although a student teacher implemented group work, it seemed not to have a particular objective and no instruction was given to pupils apart from forming groups. Any single method possesses drawbacks and may not be enough to examine what it is intended to 
measure. The use of multiple methods can strengthen construct validity, as suggested by several researchers (e.g. Yin 2014; Johnson 1997).

Continuing the discussion on the observation results, the observable practices with relatively more teacher-directed activities seemed to arise from epistemology that people in Tanzania have inherited transversally. According to Cameron and Dodd (1970), knowledge has been traditionally considered as predetermined and unquestionable, and values, beliefs and customs had to be passed on from generation to generation. This view aligns better with rationalist epistemology underpinning more teacher-centred pedagogy; reality exists independently of the knower, whose experiences and perceptions does not count as knowledge (Davis et al. 1993). In the learning settings of indigenous Tanzania, adults became knowledge possessors with authority and learners acted as knowledge recipients (Coulson 1982). Although Nyerere encouraged the practice of democracy in schools, Lema (2006) suggests that pupils' participation in curriculum planning or decision-making process did not happen. The son of Nyerere in his interview also indicated that Nyerere himself was aware of the incompatibility of democratic student-teacher relationships with Tanzanian culture. He noted:

In Tanzania, younger people have to respect the elderly. Particularly in Zanaki village where Nyerere came from [...] This tradition of Tanzania would limit the effectiveness of the student-teacher equal relationship, and Nyerere was aware of that limitation. But even so, he claimed that students should be able to challenge teachers. (Interview with Nyerere's son, 22 November 2015).

To further illustrate the ambiguities of Nyerere's ideological influence with respect to teaching practices and student-teacher relationships, data from teacher interviews implied the 
absence of Nyerere's educational legacy in current Tanzanian society. Most teachers held Nyerere and his ujamaa-related ideas in high esteem, but many admitted that present Tanzania has lost his legacy. Teacher Abdu highlighted the change of syllabi, that the selfreliant activities as well as the idea of ujamaa and socialism were absent from the present syllabi. Kito regretted Tanzania as not united anymore due to different textbooks being used across the country. On top of these perceived historical changes from Nyerere's era, a critical dimension of pedagogy was entirely absent in these teachers' accounts. The teachers were asked what they know about Nyerere, how they understood his policy ideas, and what, if any, aspects of these ideas may be reflected in today's school. No teacher brought up pedagogical approaches that Nyerere promoted in ESR, such as implementing democratic processes in decision making or practising hands-on learning and collaborative work. This observation substantiates Otunnu's $(2014,11)$ claim that ESR advocated an idealistic provision of how schools should educate children, but it was rarely implemented due to an ambiguous procedure that did not meet the educational demand of the time.

Classroom interactions manifested such transversally-inherited traditions. Few pupils raised questions to the teachers or initiated their own learning, which accounted for only $4 \%$ of all initiation moves, as opposed to $96 \%$ of teacher initiation. Teachers' checking of pupil understanding ended up as pseudo-checking, with few pupils expressing any incomprehension. Pupils frequently responded to teachers' initiation by means of whole-class responses (68\%), as if the answers were made obvious in advance. These exercises echo traditional practices of a master-learner relationship. Learners could never question or challenge the knowledge of adults and had to remember what the adults transmitted (Cameron and Dodd 1970). When such a transversally-formed cultural framework meets vertically-transmitted LCP policies underpinned by constructivist epistemology, the latter would result in a new, localised policy divergent from the original intention, as demonstrated 
by a combination of mixed methods data from observations, questionnaires, academic tests, interviews and FGDs carried out along the horizontal axis.

\section{Horizontal investigation (2): Mixed methods data}

The mixed methods data sometimes conformed but at other times contradicted one another. For instance, teachers at private schools expressed difficulty in diverging from set curricula, which pupils also talked about ('Curriculum flexibility' row in Table 2). Reports from both sides also corresponded regarding the availability of school conditions and teaching resources, with rural public schools revealing resource shortage and private schools exhibiting resource adequacy as shown in 'Teaching resources' row in Table 2. On the other hand, to a question about pupil-teacher relationships, the majority of teachers talked about positive relations, whereas many pupils regardless of school categories divulged teachers' corporal punishment (Table 2). Many expressed a fear of telling their opinions to their teachers, or even talking with them. Complaints about school activities - that some private schools did not have enough physical activities and made students study at weekends - also would not be evidenced without pupils' accounts. These cases of disagreements between pupils and teachers reflect Mitra's (2003) point that 'reality' depends on which social groups one belongs, indicating the robustness of using methodological triangulation.

Another example where mixed methods can bring an advantage over conventional quantitative or qualitative method alone pertains to the illustration purpose. The statistical analysis between observed-LCP (measured by structured observation), perceived-LCP (learner-centredness perceived by pupils, measured by the pupil questionnaire) and two types of learning outcomes (academic performance and learning attitudes, measured by the academic tests and pupil questionnaire respectively) revealed an interesting feature of the quantitative data. Regarding learning outcomes, the pupils at private schools outperformed 
those at public schools in English and mathematics exams $(t=-16.82$, df $=1,022, p<.001)$. Among public schools, urban schools achieved significantly higher test scores than rural schools $(t=13.96, \mathrm{df}=1,022, p<.001)$. Similarly for learning attitudes, private pupils compared to public pupils $\left(\chi^{2}=(3, N=1,016)=11.72 ; p<.01\right)$, as well as urban pupils compared to rural pupils $\left(\chi^{2}(3, N=1,016)=11.52 ; p<.01\right)$, significantly highly rated their learning motivation, interest, confidence, ownership and behaviour. The variability of these learning outcomes statistically associated with perceived-LCP, suggested by the Spearman rho rank order correlation for academic performance $\left(r_{s}(1,007)=.183, p<.001\right)$ and for learning attitudes $\left(r_{s}(1,003)=.153, p<.001\right)$. The higher perceived-LCP, the higher these learning outcomes. Conversely, observed-LCP showed inconsistent relationships with both types of learning outcomes. Multiple regression analyses also showed significant associations of exam scores with perceived-LCP, but not with the time spent on LCP or encouraging feedback. It is interesting to note in Table 2 that perceived-LCP and both learning outcomes showed similar tendencies along the line of socio-economic status (SES).

Such statistical results imply what is happening between several variables, and detailed accounts gathered through pupil FGDs were useful to illustrate why perceived-LCP showed significant associations with learning outcomes. They highlighted that different elements in and beyond the school compound, and not observed-LCP alone, entangle with each other to bring about both higher perceived-LCP and better learning outcomes. Private pupils reported that they had opportunities to discuss their demands with parents at home, which might have contributed to nurturing their relatively democratic attitudes when interacting with adults. More democratic child-adult relationships at home could affect how they communicated with the teachers at school, gaining the confidence to pose question in lessons and to express opinions at school meetings. Many urban public pupils, whose perceived-LCP did not statistically differ from that of their private counterparts, lived with 
families with varied attitudes towards their children. They also encountered a variety of teachers at school in terms of how they interacted with and how much they listened to the pupils. In the rural public schools, the children experienced the most rigid hierarchical relationship both with the adults at home and with the teachers at school. They lived with the least affluent material domestic conditions, which appeared to result in their voices seldom being heard by their parents. Corporal punishment and daily communication with the teachers might have led these pupils to be too frightened to speak.

Integrating the analysis from different dimensions of the pedagogical layers, the four rows with horizontal lines in Table 2 (SES, child-adult relationships, pupil-teacher relationships and perceived-LCP) indicate that perceived-LCP might be related to how people interact within society, school and classrooms. Tanzanian culture, with its underlying rationalist epistemology, has transversally cultivated a rigid social order between adults and children, which seems to have continued into contemporary society. At the same time, culture is fluid and ever changing. The horizontal comparison revealed that some pupils, especially those at private schools, experienced more equal relations with their parents. The more democratic their parents were, the more likely they were to interact with other adults democratically. Contrarily, the more rigid relations children encountered at home, such as rural public school pupils experienced, the more likely they were to keep relational distance from other adults. Therefore, Table 2 implies a consistent association between SES, childadult relationships at home and pupil-teacher relationships in school; such an association appears to be related to perceived-LCP, or how much the pupils felt that they were centred in the classroom.

The CCS framework highlights the embeddedness of the policy process within political, social and cultural particularities. The application of mixed methods along the horizontal axis allowed the study to address both exploratory and confirmatory research 
questions, to triangulate several methods to complement other methods, and to expand the quantitative results with qualitative accounts. The vertically, horizontally and transversally imbricated aspects of pedagogy explored through mixed methods have facilitated a more comprehensive and inclusive understanding of pedagogy for educational policymaking.

\section{Conclusion}

Applying the Comparative Case Study (CCS) approach, this study investigated the inextricable links between history (transversal), macro- meso- and micro-levels (vertical), and local cases (horizontal) (Bartlett and Vavrus 2017) with regards to LCP implementation in Tanzanian primary schools. Although Bartlett and Vavrus imply the usefulness and possibility of mixed methods to be practised in their CCS framework $(2017,7)$, specific guidance as to how to do so was not sufficiently given. This paper illustrates an approach to this with an empirical example of introducing mixed methods into the CCS model.

The application of mixed methods itself could show another aspect of knowledge contribution. The present study has addressed the disproportionate empirical dependence on qualitative findings (Schweisfurth 2011; Frost and Little 2014) by employing mixed methods research. Accumulating its results could triangulate methodological applications in terms of the literature, and this could enhance the validity and reliability of the overall findings made previously (Firestone 1987). Thus, the research has attempted to stretch methodological applications in the existing literature on LCP implementation in developing countries, as well as enhancing the applicability of the CCS in educational policy research. 


\section{References}

Ackers, Jim, and Frank Hardman. 2001. Classroom interaction in Kenyan primary schools.

Compare: A Journal of Comparative and International Education 31 (2): 245-261.

Bartlett, Lesley, and Frances K. Vavrus. 2014. "Transversing the Vertical Case Study: A Methodological Approach to Studies of Educational Policy as Practice.” Anthropology \& Education Quarterly 45 (2): 131-147.

Bartlett, Lesley, and Frances K. Vavrus. 2017. Rethinking Case Study Research: A Comparative Approach. New York, NY: Routledge.

Bellino, Michelle. 2016. "So that We Do not Fall again: History Education and Citizenship in 'Postwar' Guatemala.” Comparative Education Review 60 (1): 58-79.

Brandes, Donna, and Paul Ginnis. 1986. A Guide to Student-Centred Learning. Oxford: Blackwell.

Bryman, Alan. 2016. Social Research Methods. 5th ed. Oxford: Oxford University Press. Cameron, John, and William A. Dodd. 1970. Society, Schools and Progress in Tanzania. Oxford: Pergamon.

Cara, Olga. 2017. "Mixed methods in education research.” In Designing Research in Education: Concepts and Methodologies, edited by Jon Swain, 193-216. London: Sage.

Cornelius-White, Jeffrey. H. D., and Adam P. Harbaugh. 2010. "Redefining achievement.” In Learner-centred institution: Building relationships for student success, edited by Jeffrey. H. D. Cornelius-White, and Adam P. Harbaugh, 105-119. London: Sage. Coulson, Andrew. 1982. Tanzania: A Political Economy. Oxford: Clarendon. Creswell, John W., and Vicki. L. Plano Clark. 2011. Designing and Conducting Mixed Methods Research. 2nd ed. London: Sage. 
Davis, Nancy T., Jo B. McCarty, Kenneth L. Shaw, and Amal Sidani-Tabbaa. 1993.

“Transitions from Objectivism to Constructivism in Science Education.” International Journal of Science Education 15 (6): 627-636.

Dewey, John. 1916. Democracy and Education. New York, NY: Macmillan.

Firestone, William A. 1987. "Meaning in Method: The Rhetoric of Quantitative and Qualitative Research.”Educational Researcher 16 (7): 16-21.

Frost, Melanie, and Angela W. Little. 2014. “Children's Learning Practices in Ethiopia:

Observations from Primary School Classes.” Oxford Review of Education 40 (1): 91111.

Gardinier, Meg P. 2015. "Middlemen and Midwives of Reform: The in-between Worlds of Albanian Educational Policy-Makers and Professionals." Comparative Education 51 (2): 276-292.

Greene, Jennifer C., Lehn Benjamin, and Leslie Goodyear. 2001. “The Merits of Mixing Methods in Evaluation." Evaluation 7 (1): 25-44.

Greene, Jennifer C., Valerie J. Caracelli, and Wendy F. Graham. 1989. “Toward a Conceptual Framework for Mixed-Method Evaluation Designs.” Educational Evaluation and Policy Analysis 11 (3): 255-274.

Hardman, Frank, Jim Abd-Kadir, and Fay Smith. 2008. Pedagogical renewal: Improving the quality of classroom interaction in Nigerian primary schools. International Journal of Educational Development 28 (1): 55-69.

Hardman, Frank, Jan Abd-Kadir, and Audax Tibuhinda. 2012. "Reforming teacher education in Tanzania." International Journal of Educational Development 32 (6): 826-834.

Johnson, Burke R. 1997. “Examining the Validity Structure of Qualitative Research.” Education 118 (2): 282-292. 
Knight, Stephanie L., Dawn Parker, Whitney Zimmerman, and Atman Ikhlief. 2014.

"Relationship between Perceived and Observed Student-Centred Learning

Environments in Qatari Elementary Mathematics and Science Classrooms.” Learning Environments Research 17 (1): 29-47.

Lema, Elieshi. 2006. “Introduction.” In Nyerere on Education Volume II: Selected Essays and Speeches 1961-1997, edited by Elieshi Lema, Issa Omari, and Rakesh Rajani, vxiii. Dar es Salaam, Tanzania: HakiElimu.

McCombs, Barbara L., and Lynda Miller. 2007. Learner-Centered Classroom Practices and Assessments: Maximizing Student Motivation, Learning, and Achievement. Thousand Oaks, CA: Corwin Press.

Mitra, Dana L. 2003. "Student Voice in School Reform: Reframing Student-Teacher Relationships.” McGill Journal of Education 38 (2): 289-304.

Monte-Sano, Chauncey. 2008. "Qualities of Historical Writing Instruction: A Comparative Case Study of Two Teachers' Practices." American Educational Research Journal 45 (4): 1045-1079.

Mtika, Peter, and Peter Gates. 2010. "Developing Learner-Centred Education among Secondary Trainee Teachers in Malawi: The Dilemma of Appropriation and Application.” International Journal of Educational Development 30 (4): 396-404.

Mundy, Karen E., Andy Green, Bob Lingard, and Antoni Verger. 2016. "The Globalization of Education Policy - Key Approaches and Debates." In The Handbook of Global Education Policy, edited by Karen Mundy, Andy Green, Bob Lingard, and Antoni Verger, 1-20. Chichester: John Wiley \& Sons..

Author 2 .

Author 2. 
Nyerere, Julius. K. 1967. Education for Self-Reliance. Dar es Salaam, Tanzania: Government Printer.

Nyerere, Julius. K. 2004. "Progress in Schools (1967).” In Nyerere on Education, edited by Elieshi Lema, Marjorie Mbilinyi, and Rakesh Rajani, 90-94. Dar es Salaam, Tanzania: HakiElimu.

Author 2.

Onwuegbuzie, Anthony J., R. Burke Johnson, and Kathleen M. T. Collins. 2009. “A Call for Mixed Analysis: A Philosophical Framework for Combining Qualitative and Quantitative.” International Journal of Multiple Research Approaches, 3: 114-139. Otunnu, Ogenga. 2014. “Mwalimu Julius Kambarage Nyerere’s Philosophy, Contribution, and Legacies.” African Identities 1-16.

Patton, Michael Q. 2015. Qualitative Research and Evaluation Methods: Integrating Theory and Practice. 4th ed. Los Angeles, CA: Sage.

Pontefract, Caroline, and Frank Hardman. 2005. The discourse of classroom interaction in Kenyan primary schools. Comparative Education 41 (1): 87-106.

Robson, Colin. 2002. Real world research: A resource for social scientists and practitionerresearchers (2nd ed.). Oxford: Blackwell Publishing.

Rousseau, Jean-Jacques. 2007. Emile. (B. Foxlay, trans.). Champaign, Ill.: Book Jungle. (Original work published 1762).

Schweisfurth, Michele. 2011. "Learner-Centred Education in Developing Country Contexts: From Solution to Problem?” International Journal of Educational Development 31 (5): 425-432.

Sheridan, Kimberly M., Erica R. Halverson, Breanne K. Litts, Lisa Brahms, Lynette JacobsPriebe, and Trevor. Owens. 2014. "Learning in the Making: A Comparative Case Study of Three Makerspaces." Harvard Educational Review 84 (4): 505-531. 
Shriberg, Janet. 2008. "Fractured Help: Social Justice and Teacher Wellbeing in Postwar Liberia.” PhD diss., Retrieved from ProQuest Dissertations Publishing. (3327008)

Sifuna, Daniel N., and James G. Kaime. 2007. "The effect of in-service education and training (INSET) programmes in mathematics and science on classroom interaction: A case study of primary and secondary schools in Kenya." Africa Education Review 4 (1): 104-126.

Tabulawa, Richard T. 2004. "Geography students as constructors of classroom knowledge and practice: A case study from Botswana.” Journal of Curriculum Studies 36 (1): 5373.

Tabulawa, Richard T. 2013. Teaching and learning in context: Why pedagogical reforms fail in sub-Saharan Africa. Dakar, Senegal: Council for the Development of Social Science Research in Africa (CODESRIA).

Tashakkori, Abbas M., and Charles B. Teddlie. 2003. "The Past and Future of Mixed Methods Research: From Data Triangulation to Mixed Model Designs.” In Handbook of Mixed Methods in Social and Behavioral Research, edited by Abbas M.

Tashakkori, and Charles B. Teddlie, 671-701. Thousand Oaks, CA: Sage.

UNESCO. 2007. Education for All by 2015: Will We Make It? Summary. Paris: UNESCO.

UNESCO. 2017. Education for Sustainable Development Goals: Learning Objectives. Paris: UNESCO.

UNICEF. 2009. Manual: Child-Friendly Schools. New York, NY: UNICEF.

UNICEF EAPRO. (United Nations Children's Fund East Asia and Pacific Regional Office). 2006. Assessing Child-Friendly Schools: A Guide for Programme Managers in East Asia and the Pacific. Bangkok: UNICEF EAPRO. 
Vavrus, Frances K. 2009. “The Cultural Politics of Constructivist Pedagogies: Teacher Education Reform in the United Republic of Tanzania.” International Journal of Educational Development 29 (3): 303-311.

Vavrus, Frances K., and Lesley Bartlett. 2006. “Comparatively Knowing: Making a Case for the Vertical Case Study." Current Issues in Comparative Education 8 (2): 95-103.

Vavrus, Frances K., and Lesley Bartlett. 2009. "Introduction: Knowing, Comparatively.” In Critical Approaches to Comparative Education: Vertical Case Studies from Africa, Europe, the Middle East, and the Americas, edited by F. K. Vavrus, and L. Bartlett, 118. Basingstoke: Palgrave Macmillan.

Vygotsky, Lev S. 1986. Thought and Language. Translated and edited by A. Kozulin. Cambridge, MA: MIT Press.

Yin, Robert K. 2014. Case Study Research: Design and Methods. 5th ed. London: Sage. 